\title{
Organoids and epithelial ovarian cancer - a future tool for personalized treatment decisions? (Review)
}

\author{
YAGMUR SISMAN $^{1,2}$, TINE SCHNACK $^{3}$, ESTRID HØGDALL $^{2}$ and CLAUS HØGDALL ${ }^{1}$ \\ ${ }^{1}$ Department of Gynecology, Copenhagen University Hospital, Rigshospitalet, 2100 Copenhagen; \\ ${ }^{2}$ Department of Pathology, Copenhagen University Hospital, Herlev Hospital, 2730 Herlev; \\ ${ }^{3}$ Department of Gynecology, Odense University Hospital, 2100 Copenhagen, Denmark
}

Received April 9, 2021; Accepted October 14, 2021

DOI: $10.3892 / \mathrm{mco} .2021 .2462$

\begin{abstract}
Epithelial ovarian cancer (EOC) is the 5th leading cause of cancer-associated death in females worldwide. Although $80 \%$ of cases respond well to initial treatment, $>70 \%$ develop recurrent disease and become chemoresistant within the first two years. Therefore, there is a great need for predictive biomarkers to guide treatment. In the era of precision medicine, organoids are studied as a functional method to predict treatment response to oncological treatment. The overall purpose of the present systematic review was to uncover the current status of patient-derived organoids and their ability to perform drug screenings for EOC. A systematic search for studies investigating ovarian cancer and organoids was performed using PubMed and the Cochrane Library. A total of 10 studies fulfilled the inclusion criteria. The growth rates of organoids were described in six studies and varied between 29 and $90 \%$. Only four studies included data on clinical outcomes and indicated a positive correlation between clinical response and drug screening results. Inter- and intratumoral heterogeneity was examined in seven studies. They all suggested that the organoids recapture the tumor heterogeneity. Only one study performed drug screenings on organoids obtained from different tumor sites and metastasis from the same patient with EOC and revealed a different response to at least one drug for all patients. In conclusion, organoids may provide a platform for predicting the clinical response to chemotherapy and gene-targeting therapy. However, the results are only exploratory and the number of published drug screening studies is minimal. Further research is required to prove that organoids are able to support the choice of oncological treatment in patients with EOC.
\end{abstract}

Correspondence to: Dr Yagmur Sisman, Department of Gynecology, Copenhagen University Hospital, Rigshospitalet, Blegdamsvej 9, 2100 Copenhagen, Denmark

E-mail: yagmur.sisman@regionh.dk

Key words: ovarian cancer, organoids, precision medicine, drug screening, targeted therapy

\section{Contents}

1. Introduction

2. Method

3. Overview of the publications

4. Discussion

5. Conclusion

\section{Introduction}

Epithelial ovarian cancer (EOC) is the 5th leading cause of cancer-associated death in females worldwide. In $>70 \%$ of cases, it is diagnosed at an advanced stage [International Federation of Gynecology and Obstetrics (FIGO) stage III or IV], as the symptoms are frequently nonspecific $(1,2)$. Standard initial treatment is cytoreductive surgery followed by carboplatin and paclitaxel with bevacizumab as the first-line treatment of 'high-risk' patients to improve progression-free survival (PFS). Although $80 \%$ respond well to initial treatment, $>70 \%$ develop recurrent disease within the first two years and eventually become chemoresistant (2).

Treatment with bevacizumab [a monoclonal antibody that binds to vascular endothelial growth factor (VEGF inhibitor)] has improved the PFS (currently 3.3-4.0 months) in EOC, whereas no significant difference in overall survival was reported (3-11). Unfortunately, no biomarker predictive of response to bevacizumab is currently available.

Poly(adenosine diphosphate ribose) polymerase (PARP) inhibitors have changed the clinical management of EOC by targeting the homologous recombination repair pathway. PARP inhibitors are successfully implemented in treating high-grade serous ovarian carcinoma (HGSC) by leveraging inherent defects in DNA repair mechanisms presented in $\sim 50 \%$ of HGSC (12). Several trials have confirmed the positive prognostic impact of maintenance treatment in platinum-sensitive females harboring BRCA1/2 pathogenic mutations (13-19). Despite these novel treatment strategies, the leading cause of death in HGSC remains chemoresistance. Predicting response to platinum-based chemotherapy in the primary and the recurrent setting is not possible yet $(20,21)$.

In this regard, organoids, a 3-dimensional (3D) cell culture derived from stem cells, provide a novel in vitro platform to 
predict drug response. Organoids are in vitro 3D cultures grown from stem cells and consisting of organ-specific cell types (22-24). Following this definition, the first organoids were described in 2009 in murine intestinal cells, further developed for other organs, and eventually translated into human cells (25). Patients' tumor biopsies are dissociated into fragments and cells, embedded in a 3D extracellular matrix scaffold (such as Matrigel) and cultured in a cocktail of growth and signaling factors, which must be defined and optimized for each cancer type (26). Organoids have evolved through the last decade and now closely reflect primary tissue's biology and pathology, enabling their use in a broad range of applications, such as drug development and drug screening $(22,27-29)$.

Furthermore, organoids may be cultured from tumor tissue and expanded within one month. Multiple organoids may be generated from different tumor areas to mimic tumor heterogeneity $(30,31)$. Of note, tumor organoids capture inter- and intratumor heterogeneity (29). The establishment of tumor-derived organoids from various cancer types such as colon, pancreatic, gastric, prostate, breast, esophageal, bladder and endometrial cancers has been reported (30-37). The rate of successful growth of organoid cultures varies by cancer type and is highest in colon cancer, reaching $65-70 \%(28,38-42)$.

Furthermore, organoids may be used for drug screening. For instance, drug screening of a library containing $>50$ drugs was performed with 19 colorectal cancer (CRC) organoids, demonstrating a correlation between drug sensitivity and genetic aberrations in patient-derived organoids (PDOs) (43). Yan et al (32) screened 37 anticancer drugs in nine gastric cancer organoids, identifying possible responses toward novel targets. Recent studies have indicated that in-vitro drug screening using PDOs predict patient response to chemotherapy and targeted drugs in patients with CRC $(28,38)$. In EOC research, organoid cultures have been steadily emerging throughout the last years. Regular fallopian tube and ovarian surface epithelium-derived organoids that capture the genomic features of the respective tissues are established. They may offer a platform for studying cancer initiation from these potential origins (44-46). In addition to the healthy organoid lines, both short- and long-term organoid cultures from EOC have been established from several histologic subtypes (Table I). However, there are also obstacles to address in the use of PDOs. Reliable protocols are required to reproduce the tumor microenvironment, and there is currently a lack of large, well-designed prospective studies examining drug screenings regarding clinical outcomes. This may be the reason why PDOs are still on an investigational level and not routinely used in the clinic for EOC and other cancer types (Fig. 1).

The present systematic review reported on the current status of PDOs and their use in EOC to predict treatment response.

\section{Method}

The search in PubMed and the Cochrane Library was performed on July 10, 2020 (Fig. 2). The following search criteria were used: 'Ovarian Cancer', 'Organoids' and 'Cell culture techniques' (Fig. S1-S5). The literature search was combined with a screening of the reference sections of relevant studies, which did not add any new studies. The first author performed a screening of all identified articles by title and abstract. In total, 1,244 studies were screened and the full text of 123 studies was examined. Only original studies with successful EOC organoid growth and drug screening ability were included. Studies were excluded if they had included other cancer types than EOC, were not original studies or were performed on animals. Furthermore, studies in languages other than English, Danish, Swedish or Norwegian were excluded. One of the studies was included during the review process. Finally, a total of 10 studies were included in the present review (Fig. 2).

\section{Overview of the publications}

A recent study by Kopper et al (46) from 2019 published a breakthrough in EOC organoids (Table I). They presented a protocol capable of cultivating long-term organoids of all major subtypes of EOC and established 56 organoids (success rate of growth was $65 \%$ ) from 32 different patients. The organoids maintained the genomic landscape, histological aspects and tumor heterogeneity of the original tumor. Finally, they demonstrated that EOC organoids may be used for drug screening assays and capture different tumor subtype responses to standard platinum-based chemotherapy. Furthermore, organoids were tested for homologous recombination (HR) deficiency (HRD) using the recombination capacity test and it was determined that cells with HRD were sensitive to PARP inhibitors.

In another recent publication by Phan et al (47), a method of cultivating organoids derived from four patients was performed, including one patient with high-grade mixed type carcinoma with a component of HGSC (40\%) and a clear cell carcinoma $(60 \%)$, one patient with HGSC, one patient with ovarian carcinosarcoma and one patient with high-grade peritoneal carcinoma. Drug screening was achievable within one week from harvesting the original tumor. Tumor heterogeneity was preserved and multiple drugs were tested during the same period. Drug screening results reflected clinical outcomes and revealed a positive correlation; one patient had persistent disease despite aggressive debulking surgery and treatment with carboplatin and paclitaxel. Resistance to carboplatin was also observed in the high-throughput assay. Another patient was diagnosed with progressive, platinum-resistant HGSC and was heavily pretreated prior to sample procurement. The organoids from this patient were also platinum-resistant, with no reduction of viability observed upon treating the cells with carboplatin.

Maru et al (48) reported on the propagation of nine ovarian organoid cultures derived from HGSC, mucinous and endometrioid ovarian carcinoma with an overall growth success rate of $60 \%$. These organoids were long-term cultured. However, the exact duration of the cultivation was not specified. Intratumor heterogeneity was maintained and represented the original histological and genetic features. Maru et al (48) also established a drug sensitivity assay using spheroids derived from the ovarian organoids.

Jabs et al (49) harvested organoids for drug testing from 2D cell cultures of HGSC. However, these organoids were grown within 10 days. They screened cells from nine patients with HGSC with clinically relevant drugs and determined that homologous recombination deficiency scores correlated 


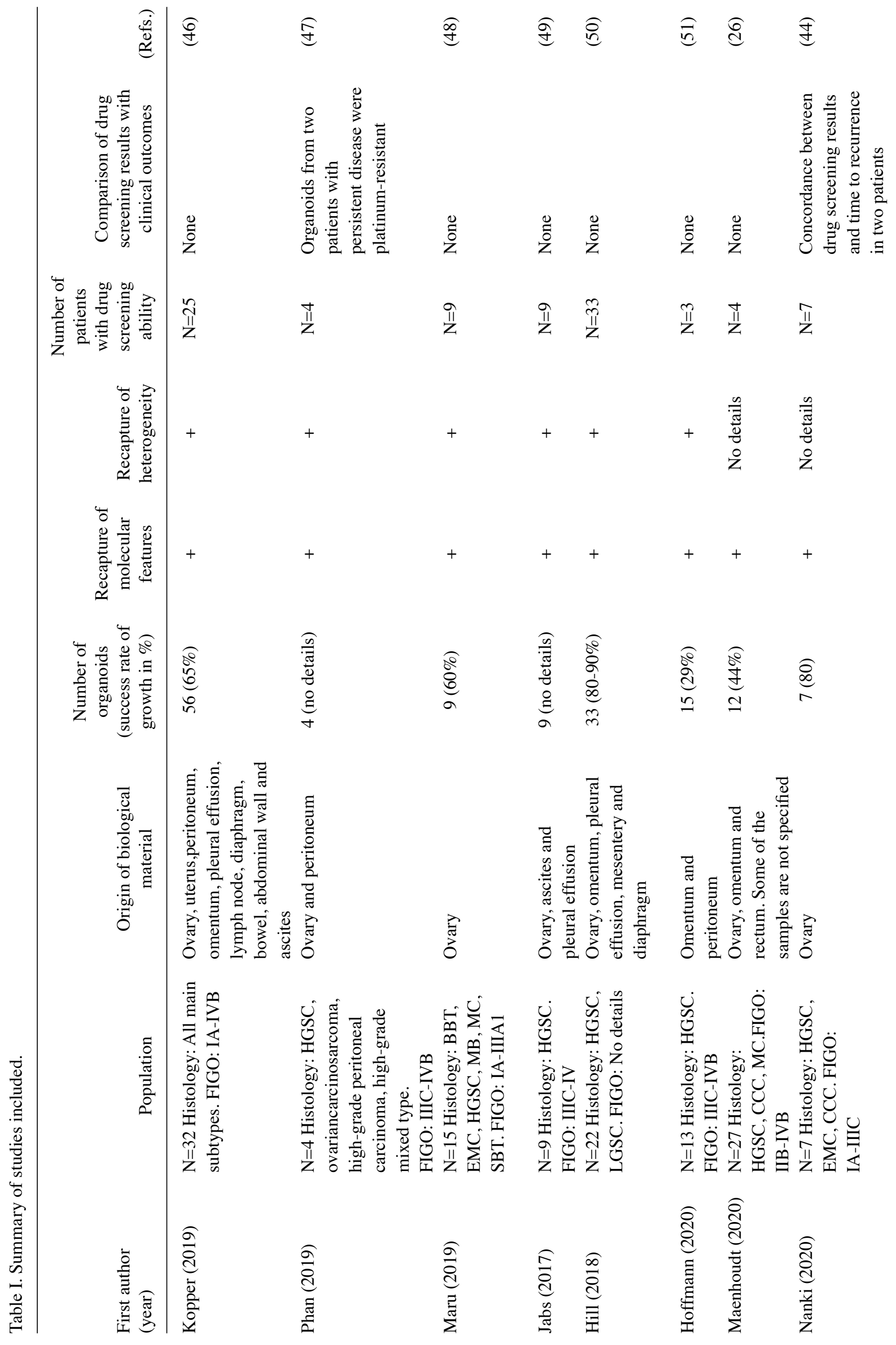




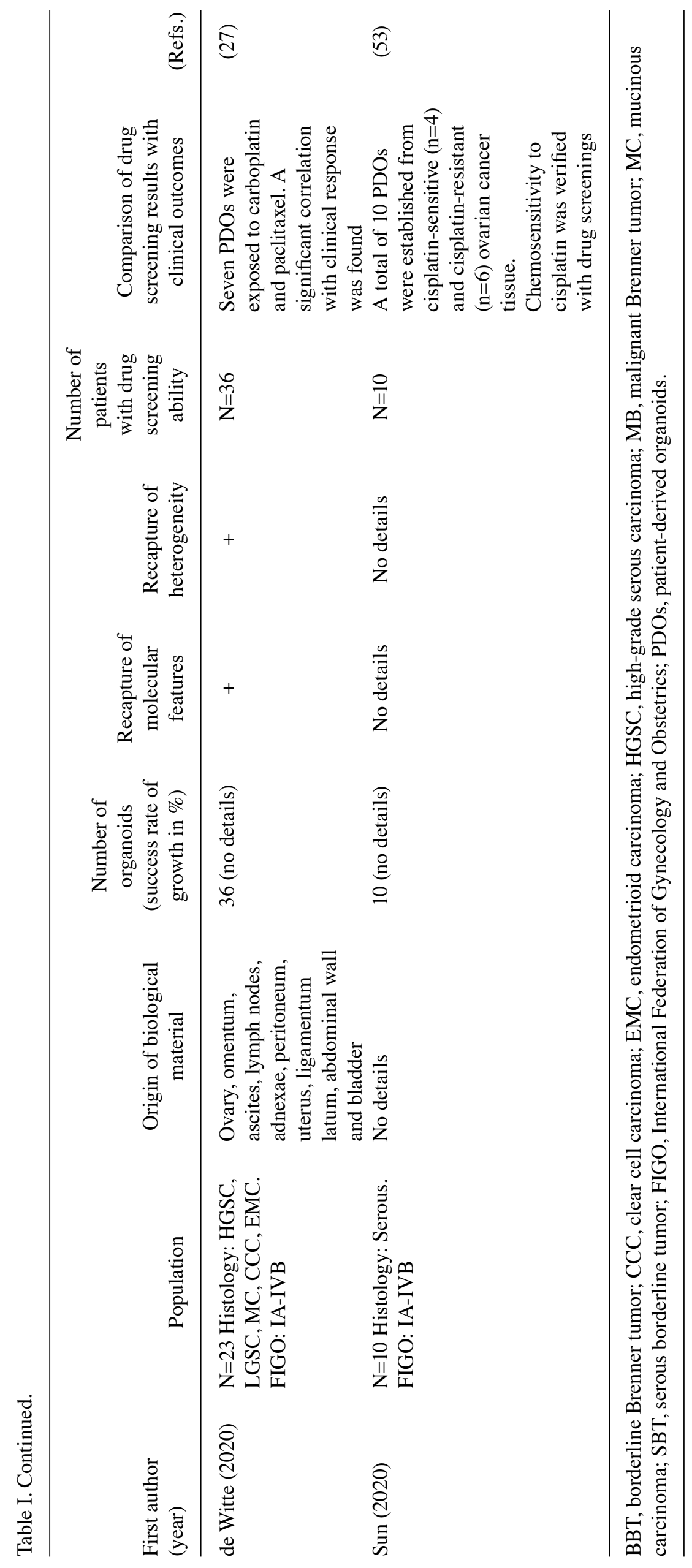




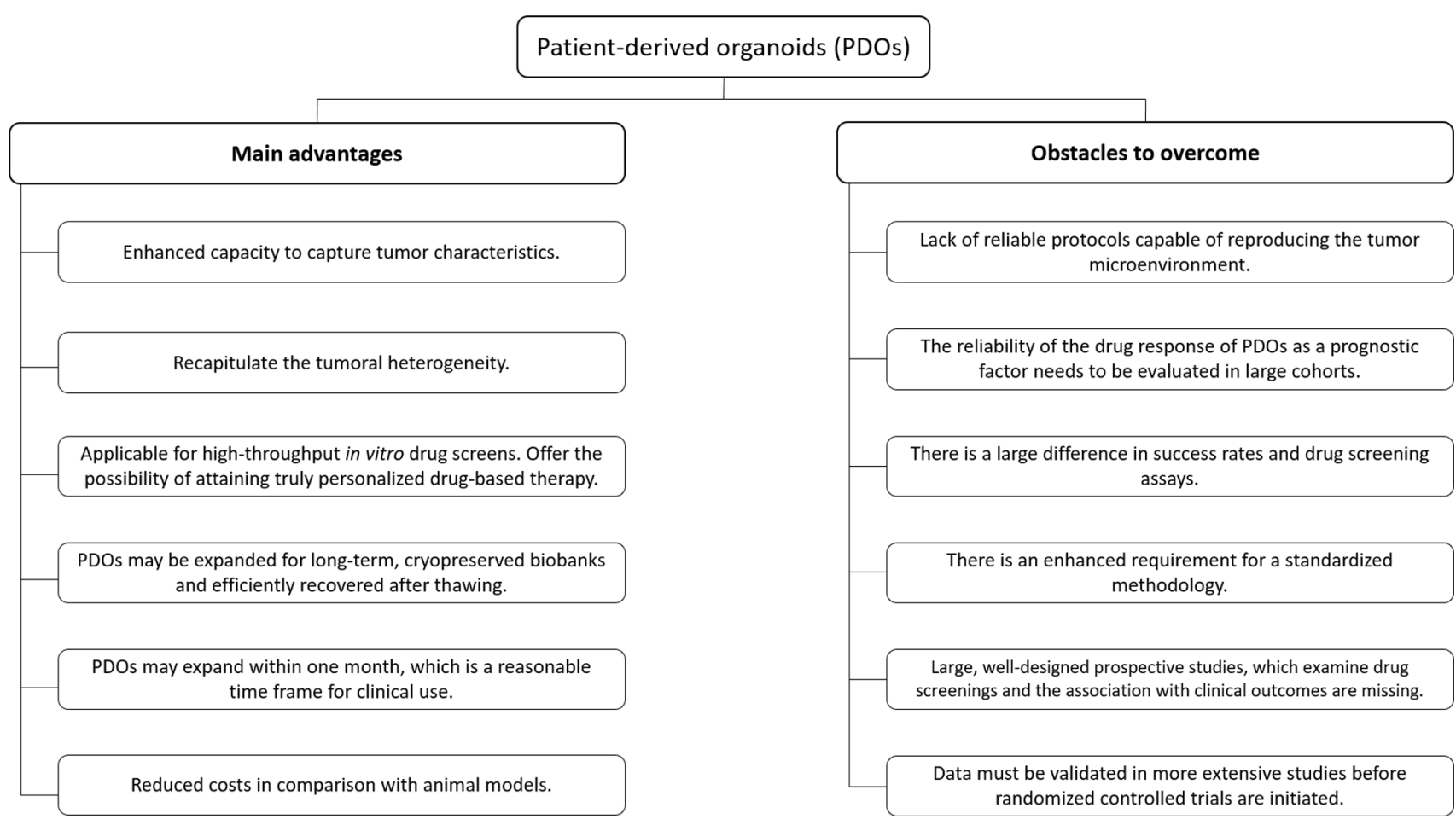

Figure 1. Main advantages and obstacles to overcome in PDOs. PDOs, patient-derived organoids.

with drug effects in organoids. The HRD score in this study was defined as the number of loss of heterozygosity regions observed in a tumor sample. HRD scores in this study varied between 3 and 22. They correlated with cytotoxic responses to carboplatin and all its combinations and paclitaxel, azacytidine and decitabine responses. However, these drugs do not directly affect DNA structure or repair. Furthermore, positive HRD scores $(\geq 10)$ determined for tumor tissues co-occurred with high drug-induced cytotoxicity and fast organoid growth.

Hill et al (50) reported on short-lived (2 weeks) HGSC organoids for drug testing. The organoids maintained their parent heterogeneity and did not develop any novel mutations. This group used organoids to assess DNA damage repair defects and their influence on immune-oncologic agents, apparently providing a possible tool to predict patient response to therapy. By testing the HR defects of 33 organoids (growth success rate $80-90 \%$ ) in 22 patients, the study confirmed that HRD in the organoids is related to sensitivity to PARP inhibitors regardless of the mutation status of DNA repair genes.

Hoffmann et al (51) created 15 organoids (growth rate, 29\%) from 13 primary deposits from patients with advanced HGSC. They indicated that the mutational and phenotypic profile of the organoids closely matched those of the parental tumor. Preliminary tests with carboplatin indicated individual differences in drug response of organoids from three patients.

Maenhoudt et al (26) established organoid cultures of tissue from patient-derived EOC, particularly from HGSC. A total of 27 patients were included and twelve organoids were established. The overall growth rate was $36 \%$ for patients with HGSC and $44 \%$ for all patients. The organoids established exhibited tumor-associated morphology and disease characteristics and recapitulated the parent tumors' marker expression and mutational landscape. Furthermore, the organoids displayed tumor-specific sensitivity to clinical chemotherapeutic drugs.

Nanki et al (44) established seven patient-derived EOC organoids in $<3$ weeks with a growth rate of $80 \%$. These organoids captured the characteristics of histological cancer subtypes and replicated the mutational landscape of the primary tumors. Seven pairs of organoids (three HGSC, one clear cell, three endometrioid) and original tumors shared 59\% of the variants identified. Furthermore, drug screening was possible and the organoid harboring a BRCA1 mutation had a higher sensitivity to olaparib and platinum drugs than the other organoids. They also compared the time to recurrence after completion of the first-line platinum regimen against drug screening results in two patients and observed concordance between the results. Of note, one patient was sensitive to paclitaxel, docetaxel, topotecan, SN-38, gemcitabine and trabectedin, and her time to recurrence after completing the first-line platinum regimen was 18 months. The time to recurrence in another patient who exhibited resistance to all tested drugs, except trabectedin, was nine months.

de Witte et al (27) included 36 organoids [29 of which have been established previously by Kopper et al (46) from 23 patients with EOC. PDOs resembled the tumors from which they were derived, with an average overlap of $67 \%$ of single nucleotide variants and similar copy number alteration profiles. Intra-patient tumor heterogeneity assessment in seven patients with organoids derived from multiple tumor locations revealed a differential response to at least one drug for all patients. Furthermore, organoids displayed inter- and intra-patient drug response heterogeneity to chemotherapy and targeted drugs. A total of seven PDOs (derived from five patients) were exposed to carboplatin and paclitaxel combination treatment in vitro. 


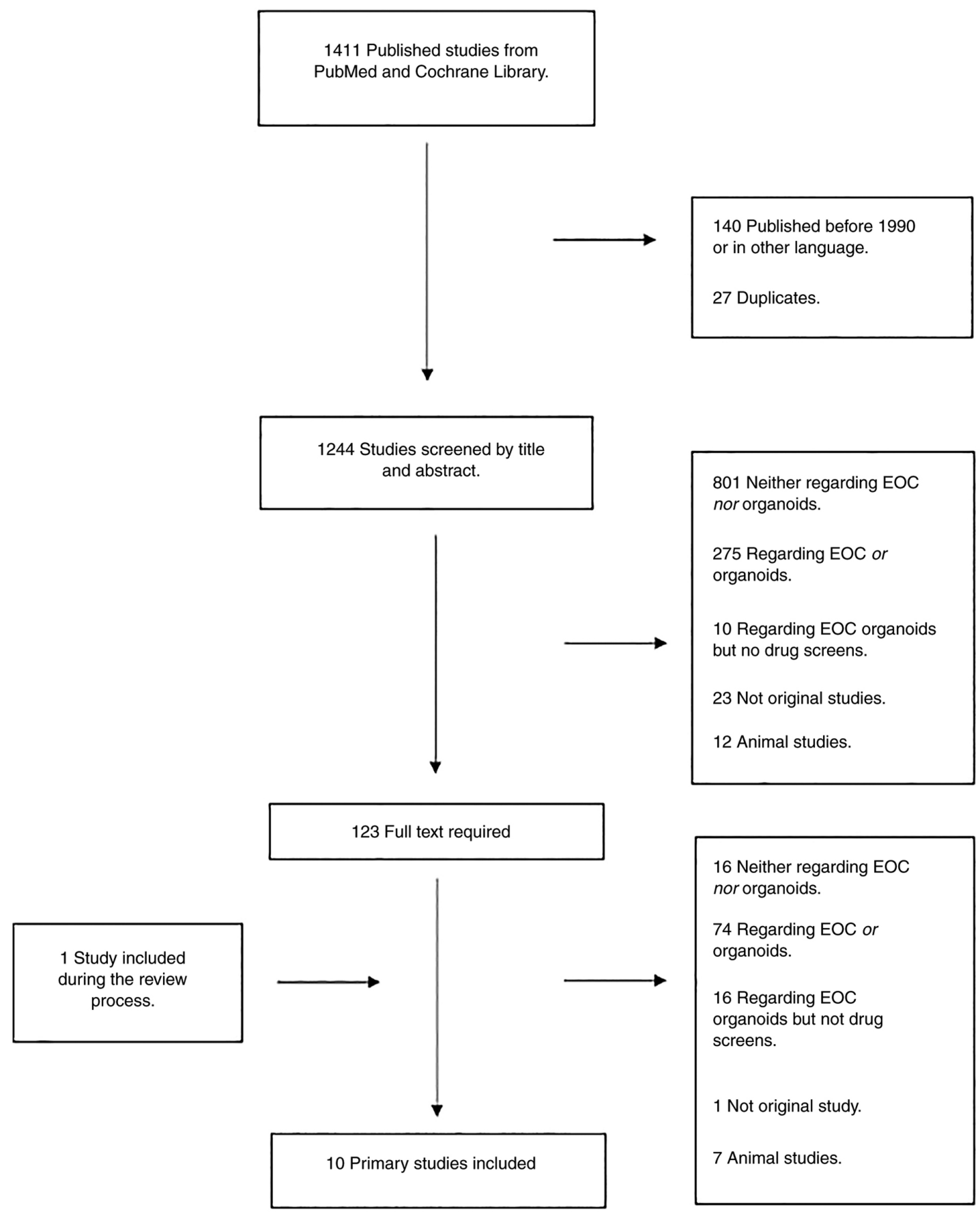

Figure 2. Flow diagram of the literature search in PubMed and Cochrane Library database made on July 10, 2020. EOC, epithelial ovarian cancer.

It was possible to directly compare their response with the patient's clinical response. These PDO drug responses had a statistically significant correlation with the clinical response, as measured by the chemotherapy response score, normalization of the serum biomarker carbohydrate antigen-125 and radiological responses [response evaluation criteria in solid tumors (RECIST)] (52). Furthermore, they demonstrated that organoid establishment and drug screening are feasible within three weeks.

Sun et al (53) established organoids $(\mathrm{n}=10)$ from cisplatin-sensitive and cisplatin-resistant ovarian cancer tissues. The PDOs verified chemosensitivity to cisplatin. RNA sequencing was employed to compare the expression of chemosensitivity-related genes in four cisplatin-sensitive and six cisplatin-resistant PDOs. Significantly higher expression levels of Aurora-A were observed in PDOs from cisplatin-resistant patients.

\section{Discussion}

In recent years, there has been an increased interest in EOC organoids, reflected in a large number of published studies. The present review aimed to uncover the current status of PDOs and their ability to perform drug screenings in EOC and thereby predict treatment response. A total of 10 studies fulfilled the inclusion criteria for this review. In all studies, 
attempts to grow EOC-organoids were successful, which were able to recapture the genomic and mutational profile and heterogeneity of the donor tissue, and drug screenings were performed $(26,27,44,46-52)$.

Other recent reviews have examined the use of PDOs in EOC $(54,55)$. A total of two studies focused on EOC, also included in the present review $(27,47)$. Both reviews concluded that PDOs were able to predict treatment response and may guide therapeutic decisions in the future. Yet, they also concluded that PDOs require to be generated and expanded efficiently to enable drug screening in a clinically meaningful time window. In addition to these reviews, the present study discussed how the growth rates may be improved and whether the histological subtypes may have a role in this context. Furthermore, the lack of clinical outcomes in numerous studies included was addressed, which is critical for the clinical use of PDOs.

Data are still limited, which is reflected by the small number of studies and number of patients. Furthermore, there is a considerable variation in the reported growth rates (29-90\%) and in four studies, the growth rates were not stated (Table I). The number and success rates of organoids used for drug screens were not reported in any of the studies included. Furthermore, there are differences in histological diagnoses. While the studies by Kopper et al (46), Maru et al (48) and de Witte et al (27) comprised all major histological subtypes of EOC, Hoffmann et al (51) and Jabs et al (49) only included patients with HGSC (Table I). At the same time, none of the studies examined the differences in growth between the different histological subgroups. The number of tissue samples was unknown and the histological subgroups were too small for a conclusive statistical analysis (46). It is well known that EOC consists of several distinct subtypes that differ in their clinical and molecular profile and should be considered and treated as uniform entities in clinical and research settings (2). Thus, optimally, organoid studies should obtain a homogenous study population by including specific subtypes of EOC to optimize growth conditions and growth rates by adapting growth medium according to histologic subtypes. Comparing these results with organoid studies from patients with gastrointestinal cancers, it was indicated that the growth rate of organoids was consistently $65-70 \%$ in these studies $(28,38-42)$. Furthermore, success rates of drug screenings (25-80\%) were mentioned and causes of failed organoid growth, such as no or few tumor cells in the biopsies, quality control problems or bacterial infections, were addressed. Clinical parameters such as sex, biopsy location and prior systemic treatment were also examined and it was determined that they did not influence the success of culture. However, even in CRC, where the use of organoids is included in clinical studies, there is still a substantial fraction of patients for whom no organoid-informed decision may be made. Growth rates may be further improved by obtaining multiple core biopsies, together with a pathologist's direct evaluation of the biopsies to identify samples with low cellularity, as suggested by Vlachogiannis et al (28).

One of the explanations for the higher growth rates observed in gastrointestinal cancers may be that they are morphologically more homogenous than EOC $(56,57)$. Only one study on pancreatic cancer stated the histopathological diagnoses as adenocarcinomas, accounting for $98 \%$ of cases (42). The growth media used in all EOC studies consisted mainly of the same components such as noggin, R-spondin, EGF, Wnt, antibiotics and ROCK inhibitor (Table SI). However, minor differences in components, such as insulin, hydrocortisone and $\beta$-estradiol, exist. The study by Maru et al (48) is the only one that has attempted to optimize the growth medium. Their initial growth rate was $45 \%$, which rose to $83 \%$ after introducing a seven-minute digestion step with Accumax, a potent proteolytic and collagenolytic enzyme with DNAse activity, following the routine digestion of tumors with dispase and collagenase. This growth rate is comparable to the growth rate of $80 \%$ obtained by Nanki et al (44). Compared with the growth medium used by Kopper et al (46), heregulin $\beta-1$, nicotinamide, forskolin, hydrocortisone and estradiol were replaced by gastrin and insulin-like growth factor. These examples highlight that growth rates may be improved with growth media modifications and emphasize the requirement for medium optimization in EOC.

Another major limitation of the reviewed studies is that only four included data on clinical outcomes $(27,44,47,53)$. All studies indicated a positive correlation between clinical response and drug screenings. de Witte et al (27) exposed seven PDOs to carboplatin and paclitaxel and determined a significant correlation with clinical response. Nanki et al (44) found concordance between drug screening results and time to recurrence in two patients tested for drugs, including paclitaxel, docetaxel and olaparib. Phan et al (47), using PDOs from two patients with persistent disease, indicated that the cancers were platinum-resistant, in concordance with Sun et al (53). PDOs were established from platin-sensitive and platin-resistant patients and their chemosensitivity was verified $(44,47)$. However, none of the studies mention the number of organoids, which failed to perform drug screens.

Furthermore, the number of drug-screened organoids is minimal and thus, the results are only exploratory and require to be validated in larger studies. In gastrointestinal cancers, various studies have compared PDO drug screenings with clinical outcomes in primary and recurrent settings $(28,38,42,58,59)$. Results from these studies suggested that PDOs mainly recapitulate patient response to treatment. Only in one study, the results of drug screens did not correlate for one out of three chemotherapeutics (38). A multicenter cohort study on metastatic breast, colon and non-small cell lung cancers is being conducted by the Foundation Hubrecht Organoid Technology (TUMOROID trial) in the Netherlands. This study is ongoing and has so far indicated a positive correlation between drug response in organoids and the clinical response of patients according to the RECIST criteria (60-62). The small number of studies and cases of EOC and the fact that the studies did not compare drug responses with clinical outcomes in a standardized manner are prone to type two errors, yielding insignificant and inconclusive results. Furthermore, it raises concerns about publication bias, as studies with no correlation between drug screens and clinical outcomes may not be published. These parameters and the lack of information may give a misleading picture of the organoids' ability to predict the treatment effect of chemotherapy and targeted therapy and thus overestimate their success in a clinical context.

A high degree of inter- and intratumor heterogeneity has been described even within specific subtypes of EOC, which 
means that the molecular profile may be different, depending on the location of the tumor sample (61). Thus, molecular analysis of a single biopsy from a tumor may not represent other tumor parts. Therefore, treatment based on analysis from a single biopsy may have limited benefit as molecular pathways active in other tumor parts will lead to tumor growth. Inter- and intratumor heterogeneity was examined in seven studies (Table I). Only one study performed drug screenings on organoids obtained from different tumor sites and metastasis from the same patients. They derived two to four PDOs from distinct cancer lesions in seven patients and revealed a differential response to at least one drug for all patients (27). In CRC and pancreatic cancer, patients' organoids were established from multiple locations from tumor and metastasis. It was reported that responses to individual agents differed substantially between lesions in a single patient, which further complicates the use of PDOs to predict treatment response $(38,58)$.

Another limitation of the studies included is the lack of uniform statistical analyses. A total of four studies lacked statistical analyses $(44,47,48,50)$, while three studies use one-way ANOVA to calculate significant variation between two experimental groups $(44,49,53)$. However, none of these three studies reported the F-values, which is critical in an ANOVA test, as it tells whether the difference in the two groups is significant.

Other factors such as biopsy localization and size may also have an impact on organoid growth. In addition, the time from tissue sampling to arrival and handling in the laboratory, including the time stored refrigerated, may also impact the growth rates. None of the studies mentioned these circumstances.

In addition, large, well-designed prospective studies examining standardized organoid-based drug screenings regarding relevant clinical outcomes are currently lacking but urgently required prior to the implementation of drug screenings based on resistance patterns of organoids in a clinical setting (52). Indeed, standardization of drug screenings and transparency in all stages from growth to drug screenings is needed. This method must meet the quality criteria for a good test, such as reproducibility, consistency, validity, cost-effectiveness, non-invasiveness, and high sensitivity and specificity. So far, the most robust empirical demonstration of the utility of a medical test is a properly designed randomized controlled trial. Therefore, the already published data must be validated more extensively in future studies before randomized controlled trials with power are initiated in a clinical setting (Fig. 1). Another factor of importance in future studies is the time frame from tissue sampling to drug screenings. These results should be available prior to relevant oncological treatment in a clinical setting (62). At present, sequencing results and molecular profiles are available within few days. Therefore, PDO-based drug screens performed within a few weeks to supplement molecular analyses should be mandatory.

In addition to drug screens, organoids may also provide valuable insight into the pathogenesis of EOC. The reported high genetic stability of healthy organoid cultures over a long period enables the study of mutagenic processes in detail $(39,63)$. Particularly the role of fallopian tube epithelium and ovarian surface epithelial cells in the development of
EOC is yet to be fully elucidated (64). In the future, organoid biobanks developed from cancer patients' tissues may be a powerful tool to enforce organoids and improve the possibility of validating drug screening results. A combined effort of the US National Cancer Institute, Cancer Research UK, the UK Wellcome Trust Sanger Institute and the foundation of Hubrecht Organoid Technology, Netherlands-known as the Human Cancer Models Initiative-is ongoing to generate a large, globally accessible bank of novel cancer cell culture models, including organoids, available for the research community (39). This library of cultures and corresponding clinical data is created to aid basic research, find leads for new compounds and help explore novel therapeutic strategies (26). Organoids may be an attractive tool for developing precise treatment strategies. They are already used to select optimal therapy in a metastatic setting for gastrointestinal cancers and may be used as a future platform for EOC drug testing as well. The major advantages of organoids are clonality, the possibility for high-throughput screening and reduced cost compared to animal models (22) (Fig. 1).

Currently, clinical diagnostic molecular approaches such as next-generation sequencing are used to guide treatment decisions based on mutations and fusions that may be biologically targeted. While it remains challenging to predict the response of treatment, organoids may add essential knowledge regarding treatment response and eventually help determine optimal treatments, particularly in the setting of a recurrent disease course. However, drug screening results from organoids cannot stand alone and there is still a requirement for molecular analyses such as sequencing upfront to identify druggable targets.

\section{Conclusion}

Organoid growth in EOC is possible, although growth rates vary. The grown organoids appear to recapitulate the molecular features and heterogeneity of the original tumor. They are suitable for drug screening assays but the success rate of drug screening is still low and must be improved before implementation in the clinic may be considered. Furthermore, drug screening results must be held against clinical outcomes and examined in adequately designed randomized clinical trials. Thus, standardization and transparency in all stages, from growth to drug screenings, are required. Using organoids for diagnostic insight or biobanking may be the next step forward. Combining this technology with molecular approaches may add to the current knowledge regarding the treatment of EOC in the future.

\section{Acknowledgements}

Not applicable.

\section{Funding}

No funding was received.

\section{Availability of data and materials}

Not applicable. 


\section{Authors' contributions}

YS contributed to all stages of the review, from literature search to manuscript writing and submission. $\mathrm{EH}, \mathrm{CH}$ and TS contributed as supervisors, providing guidance and evaluation of the academic work. All authors made substantial contributions to the design, interpretation of data, drafting the manuscript and revising it critically for important intellectual content. YS and TS assessed the authenticity of all raw data to ensure their legitimacy. All authors read and approved the final manuscript.

\section{Ethics approval of consent to participate}

Not applicable.

\section{Patient consent for publication}

Not applicable.

\section{Competing interests}

The authors declare that they have no competing interests.

\section{References}

1. Ferlay J, Colombet M, Soerjomataram I, Dyba T, Randi G Bettio M, Gavin A, Visser O and Bray F: Cancer incidence and mortality patterns in Europe: Estimates for 40 countries and 25 major cancers in 2018. Eur J Cancer 103: 356-387, 2018.

2. Lheureux S, Gourley C, Vergote I and Oza AM: Epithelial ovarian cancer. Lancet 393: 1240-1253, 2019.

3. Colombo N, Sessa C, du Bois A, Ledermann J, McCluggage WG, McNeish I, Morice P, Pignata S, Ray-Coquard I, Vergote I, et al: ESMO-ESGO consensus conference recommendations on ovarian cancer: Pathology and molecular biology, early and advanced stages, borderline tumours and recurrent disease. Ann Oncol 30: 672-705, 2019.

4. Chase DM, Chaplin DJ and Monk BJ: The development and use of vascular targeted therapy in ovarian cancer. Gynecol Oncol 145: 393-406, 2017.

5. Liu FW, Cripe J and Tewari KS: Anti-angiogenesis therapy in gynecologic malignancies. Oncology (Williston Park) 29: 350-360, 2015.

6. Perren TJ, Swart AM, Pfisterer J, Ledermann JA, Pujade-Lauraine E, Kristensen G, Carey MS, Beale P, Cervantes A, Kurzeder C, et al: A phase 3 trial of bevacizumab in ovarian cancer. N Engl J Med 365: 2484-2496, 2011.

7. Burger RA, Brady MF, Bookman MA, Fleming GF, Monk BJ, Huang H, Mannel RS, Homesley HD, Fowler J, Greer BE, et al: Incorporation of bevacizumab in the primary treatment of ovarian cancer. N Engl J Med 365: 2473-2483, 2011.

8. Aghajanian C, Blank SV, Goff BA, Judson PL, Teneriello MG, Husain A, Sovak MA, Yi J and Nycum LR: OCEANS: A randomized, double-blind, placebo-controlled phase III trial of chemotherapy with or without bevacizumab in patients with platinum-sensitive recurrent epithelial ovarian, primary peritoneal, or fallopian tube cancer. J Clin Oncol 30: 2039-2045, 2012 .

9. Aghajanian C, Goff B, Nycum LR, Wang YV, Husain A and Blank SV: Final overall survival and safety analysis of OCEANS, a phase 3 trial of chemotherapy with or without bevacizumab in patients with platinum-sensitive recurrent ovarian cancer. Gynecol Oncol 139: 10-16, 2015.

10. Coleman RL, Brady MF, Herzog TJ, Sabbatini P, Armstrong DK, Walker JL, Kim BG, Fujiwara K, Tewari KS, O'Malley DM, et al: Bevacizumab and paclitaxel-carboplatin chemotherapy and secondary cytoreduction in recurrent, platinum-sensitive ovarian cancer (NRG Oncology/Gynecologic Oncology Group study GOG-0213): A multicentre, open-label, randomised, phase 3 trial. Lancet Oncol 18: 779-791, 2017.
11. Pujade-Lauraine E, Hilpert F, Weber B, Reuss A, Poveda A, Kristensen G, Sorio R, Vergote I, Witteveen P, Bamias A, et al: Bevacizumab combined with chemotherapy for platinum-resistant recurrent ovarian cancer: The AURELIA open-label randomized phase III trial. J Clin Oncol 32: 1302-1308, 2014.

12. Cancer Genome Atlas Research Network: Integrated genomic analyses of ovarian carcinoma. Nature 474: 609-615, 2011.

13. Ledermann J, Harter P, Gourley C, Friedlander M, Vergote I, Rustin G, Scott C, Meier W, Shapira-Frommer R, Safra T, et al: Olaparib maintenance therapy in platinum-sensitive relapsed ovarian cancer. N Engl J Med 366: 1382-1392, 2012.

14. Moore K, Colombo N, Scambia G, Kim BG, Oaknin A, Friedlander M, Lisyanskaya A, Floquet A, Leary A, Sonke GS, et al: Maintenance olaparib in patients with newly diagnosed advanced ovarian cancer. N Engl J Med 379: 2495-2505, 2018

15. Pujade-Lauraine E, Ledermann JA, Selle F, Gebski V, Penson RT, Oza AM, Korach J, Huzarski T, Poveda A, Pignata S, et al: Olaparib tablets as maintenance therapy in patients with platinum-sensitive, relapsed ovarian cancer and a BRCA1/2 mutation (SOLO2/ENGOT-Ov21): A double-blind, randomised, placebo-controlled, phase 3 trial. Lancet Oncol 18: 1274-1284, 2017.

16. Mirza MR, Monk BJ, Herrstedt J, Oza AM, Mahner S, Redondo A, Fabbro M, Ledermann JA, Lorusso D, Vergote I, et al: Niraparib maintenance therapy in platinum-sensitive, recurrent ovarian cancer. N Engl J Med 375: 2154-2164, 2016.

17. González-Martín A, Pothuri B, Vergote I, DePont Christensen R, Graybill W, Mirza MR, McCormick C, Lorusso D, Hoskins P, Freyer $\mathrm{G}$, et al: Niraparib in patients with newly diagnosed advanced ovarian cancer. N Engl J Med 381: 2391-2402, 2019.

18. Swisher EM, Lin KK, Oza AM, Scott CL, Giordano H, Sun J, Konecny GE, Coleman RL, Tinker AV, O'Malley DM, et al: Rucaparib in relapsed, platinum-sensitive high-grade ovarian carcinoma (ARIEL2 Part 1): An International, multicentre, open-label, phase 2 trial. Lancet Oncol 18: 75-87, 2017.

19. Coleman RL, Oza AM, Lorusso D, Aghajanian C, Oaknin A, Dean A, Colombo N, Weberpals JI, Clamp A, Scambia G, et al: Rucaparib maintenance treatment for recurrent ovarian carcinoma after response to platinum therapy (ARIEL3): A randomised, double-blind, placebo-controlled, phase 3 trial. Lancet 390: 1949-1961, 2017.

20. Tsibulak I,Zeimet AG and Marth C: Hopes and failures in front-line ovarian cancer therapy. Crit Rev Oncol Hematol 143: 14-19, 2019.

21. Joo WD, Visintin I and Mor G: Targeted cancer therapy-Are the days of systemic chemotherapy numbered? Maturitas 76: 308-314, 2013.

22. Dumont S, Jan Z, Heremans R, Van Gorp T, Vergote I and Timmerman D: Organoids of epithelial ovarian cancer as an emerging preclinical in vitro tool: A review. J Ovarian Res 12: $105,2019$.

23. Maru Y and Hippo Y: Current status of patient-derived ovarian cancer models. Cells 8: 505, 2019.

24. Clevers H: Modeling development and disease with organoids. Cell 165: 1586-1597, 2016.

25. Sato T, Vries RG, Snippert HJ, van de Wetering M, Barker N, Stange DE, van Es JH, Abo A, Kujala P, Peters PJ and Clevers H: Single Lgr5 stem cells build crypt-villus structures in vitro without a mesenchymal niche. Nature 459: 262-265, 2009.

26. Maenhoudt N, Defraye C, Boretto M, Jan Z, Heremans R, Boeckx B, Hermans F, Arijs I, Cox B, Van Nieuwenhuysen E, et al: Developing organoids from ovarian cancer as experimental and preclinical models. Stem Cell Reports 14: 717-729, 2020.

27. de Witte CJ, Espejo Valle-Inclan J, Hami N, Lõhmussaar K, Kopper O, Vreuls CPH, Jonges GN, van Diest P, Nguyen L, Clevers $\mathrm{H}$, et al: Patient-derived ovarian cancer organoids mimic clinical response and exhibit heterogeneous inter- and intrapatient drug responses. Cell Rep 31: 107762, 2020.

28. Vlachogiannis G, Hedayat S, Vatsiou A, Jamin Y, Fernández-Mateos J, Khan K, Lampis A, Eason K, Huntingford I, Burke R, et al: Patient-derived organoids model treatment response of metastatic gastrointestinal cancers. Science 359: 920-926, 2018.

29. Lõhmussaar K, Boretto $M$ and Clevers $H$ : Human-derived model systems in gynecological cancer research. Trends Cancer 6: 1031-1043, 2020.

30. Sato T, Stange DE, Ferrante M, Vries RG, Van Es JH, Van den Brink S, Van Houdt WJ, Pronk A, Van Gorp J, Siersema PD and Clevers H: Long-term expansion of epithelial organoids from human colon, adenoma, adenocarcinoma, and Barrett's epithelium. Gastroenterology 141: 1762-1772, 2011. 
31. Boj SF, Hwang CI, Baker LA, Chio II, Engle DD, Corbo V, Jager M, Ponz-Sarvise M, Tiriac H, Spector MS, et al: Organoid models of human and mouse ductal pancreatic cancer. Cell 160: 324-338, 2015

32. Yan HHN, Siu HC, Law S, Ho SL, Yue SSK, Tsui WY, Chan D, Chan AS, Ma S, Lam KO, et al: A comprehensive human gastric cancer organoid biobank captures tumor subtype heterogeneity and enables therapeutic screening. Cell Stem Cell 23: 882-897. e11, 2018.

33. Gao D, Vela I, Sboner A, Iaquinta PJ, Karthaus WR, Gopalan A, Dowling C, Wanjala JN, Undvall EA, Arora VK, et al: Organoid cultures derived from patients with advanced prostate cancer. Cell 159: 176-187, 2014.

34. Sachs N, de Ligt J, Kopper O, Gogola E, Bounova G, Weeber F, Balgobind $\mathrm{AV}$, Wind $\mathrm{K}$, Gracanin $\mathrm{A}$, Begthel $\mathrm{H}$, et al: A living biobank of breast cancer organoids captures disease heterogeneity. Cell 172: 373-386.e10, 2018.

35. Li X, Francies HE, Secrier M, Perner J, Miremadi A, Galeano-Dalmau N, Barendt WJ, Letchford L, Leyden GM Goffin EK, et al: Organoid cultures recapitulate esophageal adenocarcinoma heterogeneity providing a model for clonality studies and precision therapeutics. Nat Commun 9: 2983, 2018.

36. Lee SH, Hu W, Matulay JT, Silva MV, Owczarek TB, Kim K, Chua CW, Barlow LJ, Kandoth C, Williams AB, et al: Tumor evolution and drug response in patient-derived organoid models of bladder cancer. Cell 173: 515-528.e17, 2018.

37. Girda E, Huang EC, Leiserowitz GS and Smith LH: The use of endometrial cancer patient-derived organoid culture for drug sensitivity testing is feasible. Int J Gynecol Cancer 27: 1701-1707, 2017.

38. Ooft SN, Weeber F, Dijkstra KK, McLean CM, Kaing S, van Werkhoven E, Schipper L, Hoes L, Vis DJ, van de Haar J, et al: Patient-derived organoids can predict response to chemotherapy in metastatic colorectal cancer patients. Sci Transl Med 11: eaay2574, 2019.

39. Drost $\mathbf{J}$ and Clevers $\mathrm{H}$ : Organoids in cancer research. Nat Rev Cancer 18: 407-418, 2018

40. Weeber F, van de Wetering M, Hoogstraat M, Dijkstra KK, Krijgsman O, Kuilman T, Gadellaa-van Hooijdonk CG van der Velden DL, Peeper DS, Cuppen EP, et al: Preserved genetic diversity in organoids cultured from biopsies of human colorectal cancer metastases. Proc Natl Acad Sci USA 112 13308-13311, 2015.

41. DeHaan RK, Sarvestani SK and Huang EH: Organoid models of colorectal pathology: Do they hold the key to personalized medicine? A systematic review. Dis Colon Rectum 63: 1559-1569, 2020

42. Yao Y, Xu X, Yang L, Zhu J, Wan J, Shen L, Xia F, Fu G, Deng Y, Pan M, et al: Patient-derived organoids predict chemoradiation responses of locally advanced rectal cancer. Cell Stem Cell 26: 17-26.e6, 2020

43. van de Wetering M, Francies HE, Francis JM, Bounova G, Iorio F, Pronk A, van Houdt W, van Gorp J, Taylor-Weiner A Kester L, et al: Prospective derivation of a living organoid biobank of colorectal cancer patients. Cell 161: 933-945, 2015

44. Nanki Y, Chiyoda T, Hirasawa A, Ookubo A, Itoh M, Ueno M, Akahane T, Kameyama K, Yamagami W, Kataoka F and Aoki D: Patient-derived ovarian cancer organoids capture the genomic profiles of primary tumours applicable for drug sensitivity and resistance testing. Sci Rep 10: 12581, 2020.

45. Kessler M, Hoffmann K, Brinkmann V, Thieck O, Jackisch S, Toelle B, Berger H, Mollenkopf HJ, Mangler M, Sehouli J, et al The Notch and Wnt pathways regulate stemness and differentiation in human fallopian tube organoids. Nat Commun 6: 8989, 2015.

46. Kopper O, de Witte CJ, Lõhmussaar K, Valle-Inclan JE, Hami N, Kester L, Balgobind AV, Korving J, Proost N, Begthel H, et al An organoid platform for ovarian cancer captures intra- and interpatient heterogeneity. Nat Med 25: 838-849, 2019.

47. Phan N, Hong JJ, Tofig B, Mapua M, Elashoff D, Moatamed NA, Huang J, Memarzadeh S, Damoiseaux R and Soragni A: A simple high-throughput approach identifies actionable drug sensitivities in patient-derived tumor organoids. Commun Biol 2: 78, 2019.
48. Maru Y, Tanaka N, Itami M and Hippo Y: Efficient use of patient-derived organoids as a preclinical model for gynecologic tumors. Gynecol Oncol 154: 189-198, 2019.

49. Jabs J, Zickgraf FM, Park J, Wagner S, Jiang X, Jechow K, Kleinheinz K, Toprak UH, Schneider MA, Meister M, et al: Screening drug effects in patient-derived cancer cells links organoid responses to genome alterations. Mol Syst Biol 13: 955 , 2017.

50. Hill SJ, Decker B, Roberts EA, Horowitz NS, Muto MG, Worley MJ Jr, Feltmate CM, Nucci MR, Swisher EM, Nguyen H, et al: Prediction of DNA repair inhibitor response in short-term patient-derived ovarian cancer organoids. Cancer Discov 8: 1404-1421, 2018.

51. Hoffmann K, Berger $H$, Kulbe $H$, Thillainadarasan $S$, Mollenkopf HJ, Zemojtel T, Taube E, Darb-Esfahani S, Mangler M, Sehouli J, et al: Stable expansion of high-grade serous ovarian cancer organoids requires a low-Wnt environment. EMBO J 39: e104013, 2020

52. Eisenhauer EA, Therasse P, Bogaerts J, Schwartz LH, Sargent D, Ford R, Dancey J, Arbuck S, Gwyther S, Mooney M, et al: New response evaluation criteria in solid tumours: Revised RECIST guideline (version 1.1). Eur J Cancer 45: 228-247, 2009.

53. Sun H, Wang H, Wang X, Aoki Y, Wang X, Yang Y, Cheng X, Wang $\mathrm{Z}$ and Wang $\mathrm{X}$ : Aurora-A/SOX8/FOXK1 signaling axis promotes chemoresistance via suppression of cell senescence and induction of glucose metabolism in ovarian cancer organoids and cells. Theranostics 10: 6928-6945, 2020.

54. Nero C, Vizzielli G, Lorusso D, Cesari E, Daniele G, Loverro M, Scambia G and Sette C: Patient-derived organoids and high grade serous ovarian cancer: From disease modeling to personalized medicine. J Exp Clin Cancer Res 40: 116, 2021.

55. Wensink GE, Elias SG, Mullenders J, Koopman M, Boj SF, Kranenburg OW and Roodhart JML: Patient-derived organoids as a predictive biomarker for treatment response in cancer patients. NPJ Precis Oncol 5: 30, 2021.

56. Fleming M, Ravula S, Tatishchev SF and Wang HL: Colorectal carcinoma: Pathologic aspects. J Gastrointest Oncol 3: 153-173, 2012.

57. Haeberle L and Esposito I: Pathology of pancreatic cancer. Transl Gastroenterol Hepatol 4: 50, 2019

58. Tiriac H, Belleau P, Engle DD, Plenker D, Deschênes A, Somerville TDD, Froeling FEM, Burkhart RA, Denroche RE, Jang GH, et al: Organoid profiling identifies common responders to chemotherapy in pancreatic cancer. Cancer Discov 8: 1112-1129, 2018.

59. Weeber F, Ooft SN, Dijkstra KK and Voest EE: Tumor organoids as a Pre-clinical cancer model for drug discovery. Cell Chem Biol 24: 1092-1100, 2017.

60. Aboulkheyr Es H, Montazeri L, Aref AR, Vosough M and Baharvand H: Personalized cancer medicine: An organoid approach. Trends Biotechnol 36: 358-371, 2018.

61. Klement GL, Arkun K, Valik D, Roffidal T, Hashemi A, Klement C, Carmassi P, Rietman E, Slaby O, Mazanek P, et al: Future paradigms for precision oncology. Oncotarget 7 : 46813-46831, 2016.

62. Matchar DB: Chapter 1: Introduction to the methods guide for medical test reviews. J Gen Intern Med 27 (Suppl 1): S4-S10, 2012.

63. Behjati S, Huch M, van Boxtel R, Karthaus W, Wedge DC, Tamuri AU, Martincorena I, Petljak M, Alexandrov LB, Gundem G, et al: Genome sequencing of normal cells reveals developmental lineages and mutational processes. Nature 513: 422-425, 2014

64. Drost J, van Boxtel R, Blokzijl F, Mizutani T, Sasaki N, Sasselli V, de Ligt J, Behjati S, Grolleman JE, van Wezel T, et al: Use of CRISPR-modified human stem cell organoids to study the origin of mutational signatures in cancer. Science 358: 234-238, 2017.

This work is licensed under a Creative Commons Attribution-NonCommercial-NoDerivatives 4.0 International (CC BY-NC-ND 4.0) License. 